\title{
Length Scales of Interfacial Fracture Toughness
}

\author{
Michal K. Budzik
}

Aarhus University, Denmark

Homogeneity of materials and interfaces is a concept rather than a fact. To some level, and length scale, heterogeneities are present. Taking as an example the layered materials, heterogeneities can be linked with: materials used as adherends (e.g. fibres in matrix material); bondlines/adhesive layers (if such exists, e.g. trapped air, filling particles); surfaces and interfaces (variations in roughness or surface energy). Heterogeneities can be unwanted i.e. defects, flaws, as well as a desired, e. g. fibres in composite materials or geometric features of lattice structures or auxetic materials. In structural adhesive bonding, the quality of bonding is related with homogeneous layer of the adhesive transferring the load between the adherends. Efficiency of the load transfers depends on a strong adhesion between the adhesive layer and the adherends, and as in the previous case homogeneity of surface preparation is deemed to be one of the key factors in assuring high quality of the joint. With the focus on geometry in shaping material properties we investigate a landscape of parameters responsible for the final, apparent, properties of layered materials. In specific, we analyse the effect of simple, geometrical modifications introduced to the interface region on the fracture properties. Starting with the in-the- (crack growth) -plane modifications, viz. surface patterning, and ending with the through-the-thickness pillar like geometries, a number of length scale parameters is revealed. These allows for better understanding of fracture properties and designing of such. Links are being made to a number of applications incl. adhesive bonding, wear debris generation or mechanical metamaterials. 